\title{
Kir Channel Blockages by Proflavine Derivatives via Multiple Modes of Interaction ${ }^{\mathrm{s}}$
}

\author{
Atsushi Inanobe, Hideaki Itamochi, and Yoshihisa Kurachi \\ Department of Pharmacology, Graduate School of Medicine (A.I., H.I., Y.K.), and Center for Advanced Medical Engineering and \\ Informatics (A.I., Y.K.), Osaka University, Suita, Osaka, Japan
}

Received December 8, 2017; accepted April 6, 2018

\section{ABSTRACT}

Many compounds inhibit tetrameric and pseudo-tetrameric cation channels by associating with the central cavity located in the middle of the membrane plane. They traverse the ion conduction pathway from the intracellular side and through access to the cavity. Previously, we reported that the bacteriostatic agent, proflavine, preferentially blocked a subset of inward rectifier $\mathrm{K}^{+}$(Kir) channels. However, the development of the inhibition of Kir1.1 by the compound was obviously different from that operating in Kir3.2 as a pore blocker. To gain mechanistic insights into the compound-channel interaction, we analyzed its chemical specificity, subunit selectivity, and voltage dependency using 13 different combinations of Kir-channel family members and 11 proflavine derivatives. The Kir-channel family members were classified into three groups: 1) Kir2.2, Kir3.x, Kir4.2, and Kir6.2s36, which exhibited Kir3.2-type inhibition (slow onset and recovery, irreversible, and voltage-dependent blockage); 2) Kir1.1 and Kir4.1/Kir5.1 (prompt onset and recovery, reversible, and voltage-independent blockage); and 3) Kir2.1, Kir2.3, Kir4.1, and Kir7.1 (no response). The degree of current inhibition depended on the combination of compounds and channels. Chimera between proflavine-sensitive Kir1.1 and -insensitive Kir4. 1 revealed that the extracellular portion of Kir1.1 is crucial for the recognition of the proflavine derivative acrinol. In conclusion, preferential blockage of Kir-channel family members by proflavine derivatives is based on multiple modes of action. This raises the possibility of designing subunitspecific inhibitors.

\section{Introduction}

Voltage- and ligand-gated cation channels are necessary for cell and organ physiology, and fine-tuning their functioning using medication is a therapeutic approach for treating cardiovascular and mental illnesses. A large portion of these channels, including inward-rectifier $\mathrm{K}^{+}$(Kir) channels, possess an ion conduction pathway at the axis of a 4 -fold or pseudo-4-fold rotational symmetry (Bichet et al., 2003; Hibino et al., 2010). On the membrane plane, the pathway is constrained by the ion selectivity filter and bundle crossing that sandwich the pore-dilated portion known as the central cavity. The cavity is where various therapeutic agents bind and thereby interfere with ion conduction. The corresponding cavity of the human ether-a-go-go-related gene product is known to accommodate diverse chemicals, leading to the blockage of $\mathrm{K}^{+}$efflux during the repolarization phase of cardiac action potential, the prolongation of the QT interval in an electrocardiogram, and life-threatening cardiac arrhythmias (Sanguinetti et al., 1995). Therefore, the molecular recognition process in the central cavity of various channels has drawn increasing attention.

This work was supported by the Japan Society for the Promotion of Science Grant-in-Aid for Scientific Research (B) [Grant JP17H04018]

https://doi.org/10.1124/mol.117.111377.

[S This article has supplemental material available at molpharm. aspetjournals.org.
Salt reabsorption in renal tubules is essential for the control of blood volume and pressure. A weak inward rectifier, Kir1.1, present in the apical membranes of various nephron segments, participates in the ion transport system by secreting $\mathrm{K}^{+}$into the lumen (Ho et al., 1993; Hebert et al., 2005). Inherited loss-of-function mutations in Kir1.1 cause a saltwasting nephropathy, type II Bartter's syndrome (Hebert, 2003). Gene-silencing research into Kir1.1 yielded mice showing the same phenotype (Lorenz et al., 2002), and the symptoms are consistent with those of the side effects of loop diuretics (Sica, 2011; Tamargo et al., 2014). Therefore, Kir1.1 has been recognized as a novel therapeutic target for diuretics (Lewis et al., 2009; Bhave et al., 2011).

Previously, we isolated the bacteriostatic agent proflavine as a pore blocker of the G protein-gated Kir channel, Kir3.2 (Kawada et al., 2016). Proflavine also prevented the channel activity of Kir1.1, but the drug action was obviously different from that toward Kir3.2. This suggests that the compound acts at a site other than the central cavity and the interaction yields the inhibition of ionic current flow. In this study, we examined the binding mode of proflavine and its derivatives to Kir channels by analyzing chemical selectivity, subunit specificity, and voltage dependency. The results showed that the compounds blocked the channels through multiple modes of action. In Kir1.1, in particular, only the extracellular region mediated the current blockage. These observations both suggest a strategy for designing novel diuretics and reveal 
the feasibility of designing novel compounds targeting the extracellular region of Kir channels.

\section{Materials and Methods}

Reagents. Acridine, 9-chloroacridine, 9-aminoacridine, and acridine yellow G were purchased from Sigma-Aldrich (St. Louis, MO). Quinacrine and proflavine were purchased from Tokyo Chemical Industry (Tokyo, Japan). Acriflavine, acrinol, and diphenylamine were purchased from Nacalai Tesque (Kyoto, Japan). Acridine orange and 4,4'-diaminodiphenylamine sulfate were purchased from Waldeck (Münster, Germany) and Wako (Tokyo, Japan), respectively (Supplemental Fig. 1). These compounds were dissolved in dimethylsulfoxide daily at a concentration of $100 \mathrm{mM}$ before experiments were performed.

Molecular Biology. The full length of channel subunits subjected to cRNA synthesis were as follows: rat Kir1.1 (Kondo et al., 1996), mouse Kir2.1 (Takahashi et al., 1994), mouse Kir2.2 (Takahashi et al., 1994), mouse Kir2.3 (Morishige et al., 1994), mouse Kir3.1 (Inanobe et al., 1995), mouse Kir3.2c (Inanobe et al., 1999b), mouse Kir3.2d (Inanobe et al., 1999a), rat Kir3.4 (Krapivinsky et al., 1995), rat Kir4.1 (Takumi et al., 1995), and mouse Kir6.2, in which 36 amino acids had been deleted from the $\mathrm{C}$ terminal (Kir6.2 336 ), as well as human Kir2.4, Kir4.2, Kir5.1, and Kir7.1 (provided by the Kazusa DNA Research Institute, Chiba, Japan). Chimeras between acrinolsensitive Kir1.1 and acrinol-insensitive Kir4.1 were generated using primer-based polymerase chain reaction, and their sequences were then confirmed using Sanger sequencing, with assistance from the Center for Medical Research and Education, Osaka University.

Two-Electrode Voltage Clamp Experiments. Oocytes were surgically isolated from the abdomen of anesthetized Xenopus laevis and defolliculated using collagenase type I (Gibco; Thermo Fisher Scientific, Waltham, MA). The expression plasmids were linearized and transcribed in vitro using mMESSAGE mMACHINE Transcription Kits (Thermo Fisher Scientific). Each of the cRNAs (1.5-5 ng) was injected into the oocytes. During expression of the G-protein-gated Kir channels (in combinations of Kir3.1, Kir3.2c, Kir3.2d, and Kir3.4), the $\mathrm{G}$ protein $\mathrm{G} \beta 1$ and $\mathrm{G} \gamma 2$ subunits were also expressed, to measure the full activity of the channels without further stimulation. Heteromeric Kir4.1/Kir5.1 was reconstituted by injecting a mixture of Kir4.1 cRNA $(0.5 \mathrm{ng})$ and Kir5.1 cRNA (4.5 ng). No current was recorded from cells injected with the same amount of Kir5.1 cRNA alone. One to two days after injection of the cRNAs, the oocytes were studied using twoelectrode voltage clamp experiments at ambient temperature (22$26^{\circ} \mathrm{C}$ ), using a GeneClamp 500 amplifier (Molecular Devices, San Jose, $\mathrm{CA})$. The glass electrodes had a resistance of 0.4-1.2 $\mathrm{M} \Omega$ when filled with $3 \mathrm{M} \mathrm{KCl}$. The bath solution contained $40 \mathrm{mM} \mathrm{KCl,} 50 \mathrm{mM} \mathrm{NaCl}$, $3 \mathrm{mM} \mathrm{MgCl}_{2}, 5 \mathrm{mM}$ HEPES, and $150 \mu \mathrm{M}$ niflumic acid (pH 7.35 with $\mathrm{KOH}) . \mathrm{Ba}^{2+}$ is a general blocker for Kir channel members, and honey bee toxin, tertiapin, is a selective blocker for a subset of the members including Kir1.1 (Jin and Lu, 1998). To detect the leak current level of oocytes, $\mathrm{Ba}^{2+}(3 \mathrm{mM})$ was administered at the end of the recording. However, while Kir1.1 blockage by $\mathrm{Ba}^{2+}$ is weak at depolarization, the blockage by tertiapin is constant over voltage. Therefore, to estimate the leak current level of oocytes expressing Kir1.1 at depolarization, tertiapin $(3 \mu \mathrm{M})$ was used instead of $\mathrm{Ba}^{2+}$. Membrane-impermeable buffer 4-morpholineethanesulfonic acid and HEPES were used to reveal the effects of external $\mathrm{pH}$.

The dose-response curves for inhibition of Kir channels were fitted with Hill's equation:

$$
I(D)=\min +(\max -\min ) /\left[1+\left(D / \mathrm{IC}_{50}\right)^{n_{H}}\right]
$$

where $I$ is the relative current at the end of the test pulse, in the presence of the compound at the tested concentration $(D)$; $\mathrm{IC}_{50}$ is the half-maximal concentration of the compound; $n_{\mathrm{H}}$ is the Hill coefficient; and min and max are the minimum and maximum values, respectively, where in most situations min is 0 and max is 1 . Data acquisition and fitting were carried out using the Clampfit software (Molecular
Devices) and SigmaPlot (Systat Software, Chicago, IL). Data are presented as the mean \pm S.D. with $n$ (number of observations). Statistical analyses were performed using one-way analysis of variance with post-hoc Tukey-Kramer tests.

\section{Results}

Characteristics of Kir1.1 Blockage by Proflavine. The $\mathrm{K}^{+}$current recorded from oocytes expressing Kir1.1 was elicited by administering a combined voltage pulse. Perfusion with proflavine $(300 \mu \mathrm{M})$ decreased the current amplitude of Kir1.1 (Fig. 1A, upper panel) (Kawada et al., 2016). This inhibition developed rapidly, with a time constant of $4.3 \pm 1.7$ seconds $(n=9)$. Perfusion with a proflavine-free solution promptly and fully recovered the current amplitude (Fig. 1A, lower panel). Proflavine also blocked Kir3.2 (Fig. 1B, upper panel) (Kawada et al., 2016). However, in this case current inhibition developed slowly, with a time constant of $23.7 \pm 16.4$ seconds ( $n=10$; Fig. 1B). Recovery from this blockage was also slow, and the amplitude was not fully restored even by prolonged perfusion with a proflavine-free solution. Since proflavine did not alter the endogenous current properties of control oocytes (Supplemental Fig. 2), the rate of solution exchange in the chamber could be estimated based on the time constant of current blockage by $\mathrm{Ba}^{2+}: 1.30 \pm 0.4$ seconds $(n=48)$. We, therefore, conclude that the development of current blockage may reflect the process of reaching the binding equilibrium between proflavine and Kir channels, and also that blockage of Kir1.1 by proflavine is significantly faster than that of Kir3.2.

While proflavine mainly associates with the central cavity of Kir3.2 and attenuates its channel activity, it also behaves as an antagonist of the $\mathrm{m}_{2}$-muscarinic receptor (Kawada et al., 2016). After perfusion of proflavine, oocytes turned intensely yellowish and the color was preserved even with overnight immersion of compound-free solution. Therefore, the compounds that are retained in the cells may account for the sustained blockage of Kir3.2 activity. In contrast, the action of proflavine on Kir1.1 (rapid onset and recovery, together with reversibility of current blockage) was revealed when the drug was present in the external solution, which suggests that the compound interacts with the extracellular side of Kir1.1 and then blocks channel activity.

Compound Specificity of Kir1.1 Blockage. We then investigated whether proflavine analogs selectively block Kir1.1. The current in oocytes expressing Kir1.1 was measured by changing the membrane potential as described previously. The compounds $(100 \mu \mathrm{M})$ were administrated to the cells over a short period (0.5-1 minutes) (Fig. 2, A and C). Of the 10 proflavine analogs tested (Supplemental Fig. 1), two (acrinol and acriflavine) were found to suppress Kir1.1 current amplitude (Fig. 2, A and B). They resulted in rapid onset and recovery of the inhibition, and reversible inhibition, similar to proflavine (the time constants for the onset of acrinol, acriflavine, and proflavine were $3.4 \pm 1.4,2.9 \pm 0.9$, and $4.1 \pm 2.0$ seconds, respectively; $n=$ 6 ). The other eight compounds (acridine, acridine orange, 9-chloroacridine, 9-aminoacridine, quinacrine, acridine yellow $\mathrm{G}$, diphenylamine, and 6,6'-diaminodiphenylamine) did not exhibit inhibitory effects on Kir1.1 (Fig. 2C). Although these compounds share a basic structural unit, there were profound differences in their inhibitory effect on Kir1.1 activity, suggesting that the preference is present in the compound/Kir1.1 interaction. 
A

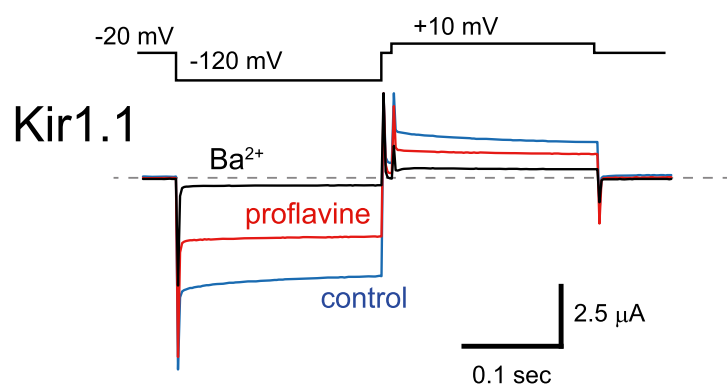

Number of sweeps

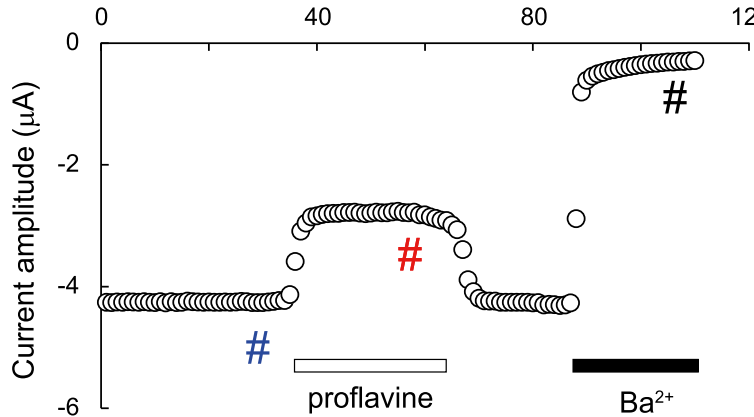

B

Kir3.2

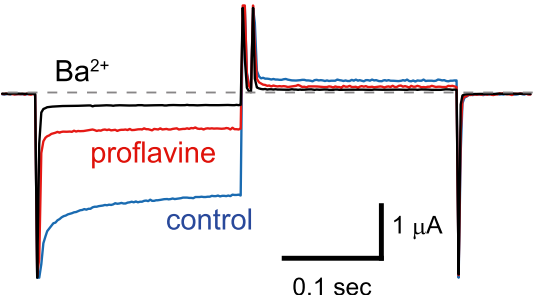

Number of sweeps

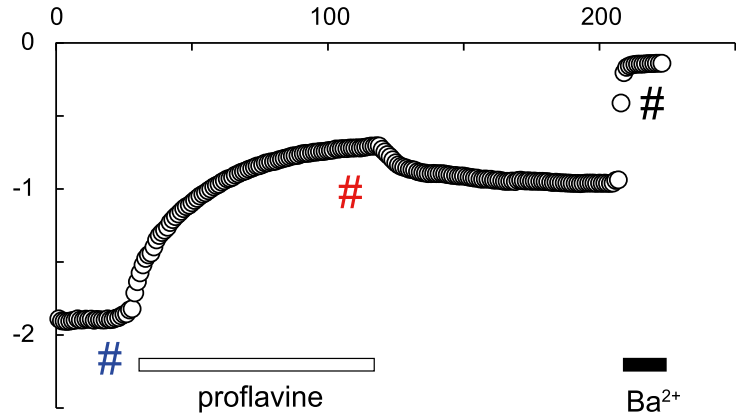

Fig. 1. Difference in current inhibition in Kir1.1 and Kir3.2 by proflavine. Currents were recorded by two-electrode voltage clamp experiments in oocytes expressing either Kir1.1 (A) or Kir3.2 (B). A combined step pulse ( $-120 \mathrm{mV}$ for 0.2 seconds and $+10 \mathrm{mV}$ for 0.2 seconds with a 10 -millisecond interval at $-20 \mathrm{mV}$ ), illustrated over a set of current traces, was delivered every 3 seconds from a holding potential of $-20 \mathrm{mV}$. The external solution contained $40 \mathrm{mM} \mathrm{K}$. The current amplitude at the end of the hyperpolarized test pulse was measured. Actual current traces, indicated by \# in the lower panels, were picked up. White and black bars indicate the periods of perfusion of proflavine $(300 \mu \mathrm{M})$ and $\mathrm{Ba}^{2+}(3 \mathrm{mM})$, respectively. Zero current is represented by the dashed line. In the oocytes expressing Kir3.2, $\mathrm{G}$ protein $\mathrm{G} \beta 1$ and $\mathrm{G} \gamma 2$ subunits were also expressed, for the constitutive activity of the channel.

Inhibition of $\mathrm{K}^{+}$Currents by Proflavine Derivatives. We then tested the effects of proflavine analogs on the Kirchannel family members. The channels were expressed in

A

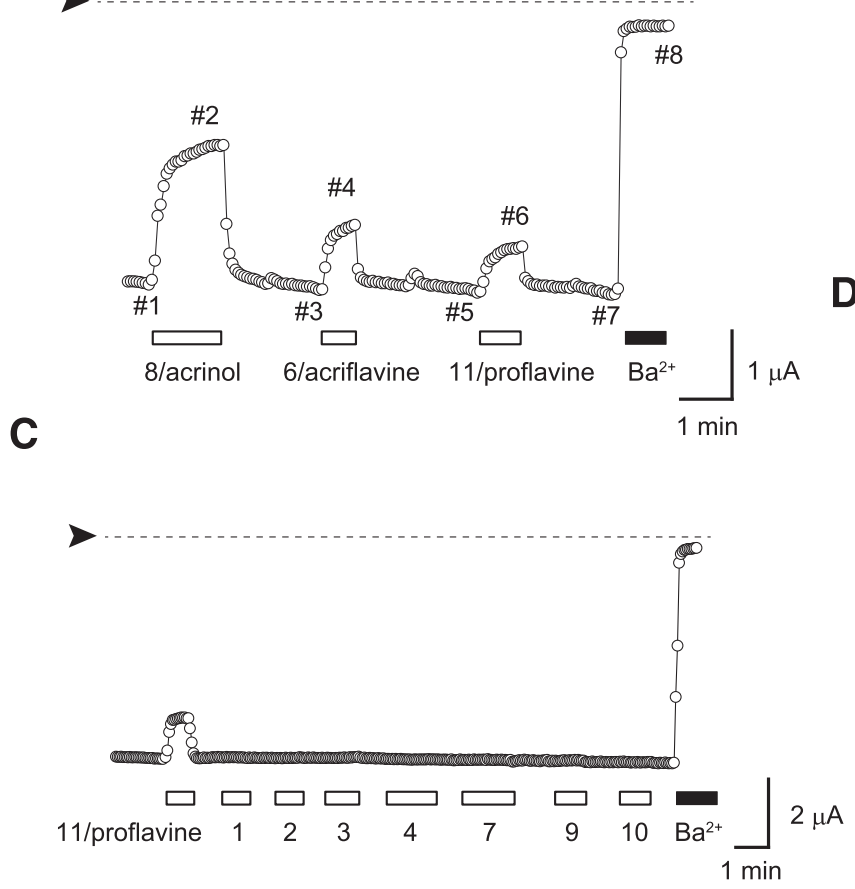

B

D oocytes by injecting various combinations of cRNAs to reconstitute classic inward rectifiers (Kir2.1, Kir2.2, Kir2.3, and Kir2.4), G protein-gated Kir channels (Kir3.1 and Kir3.2,
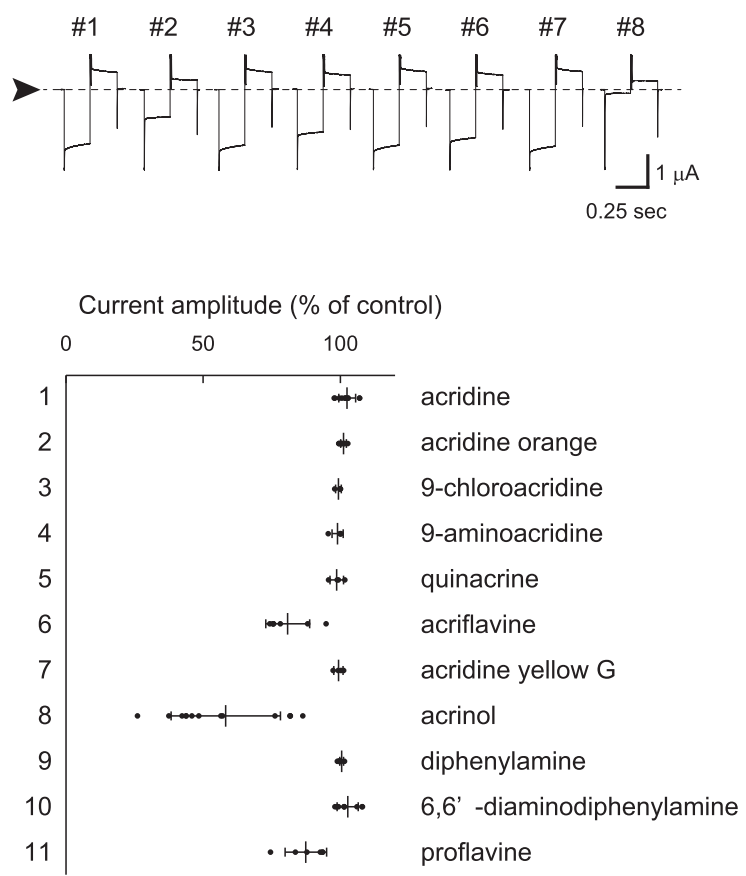

Fig. 2. Selective blockage of Kir1.1 by proflavine derivatives. (A and C) Current inhibition in Kir1.1 by proflavine analogs. Current amplitude at the end of the hyperpolarized membrane potential was obtained as described in Fig. 1. Eleven proflavine derivatives (D, numbers 1-11) were perfused at a concentration of $100 \mu \mathrm{M}$ to oocytes expressing Kir1.1. Current inhibition was observed in solutions containing acrinol (8), acriflavine (6), or proflavine (11). Actual current traces, marked by \#1-\#8 in (A), are shown in (B). (D) Summary of current blockage by proflavine analogs. Ba ${ }^{2+}$-sensitive current amplitudes in the presence of $100 \mu \mathrm{M}$ of the proflavine analog were normalized against those in its absence. Data are shown as mean \pm S.D. $(n=6-8)$. 
A

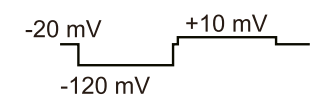

Kir4.1/Kir5.1

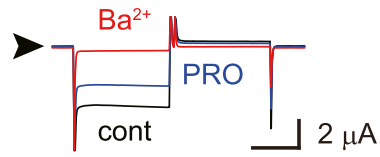

Kir3.1/Kir3.4

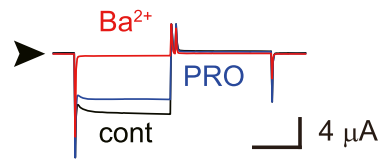

Kir2.2

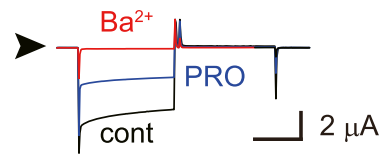

Kir2.3

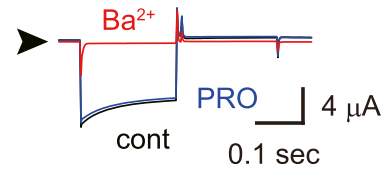

B
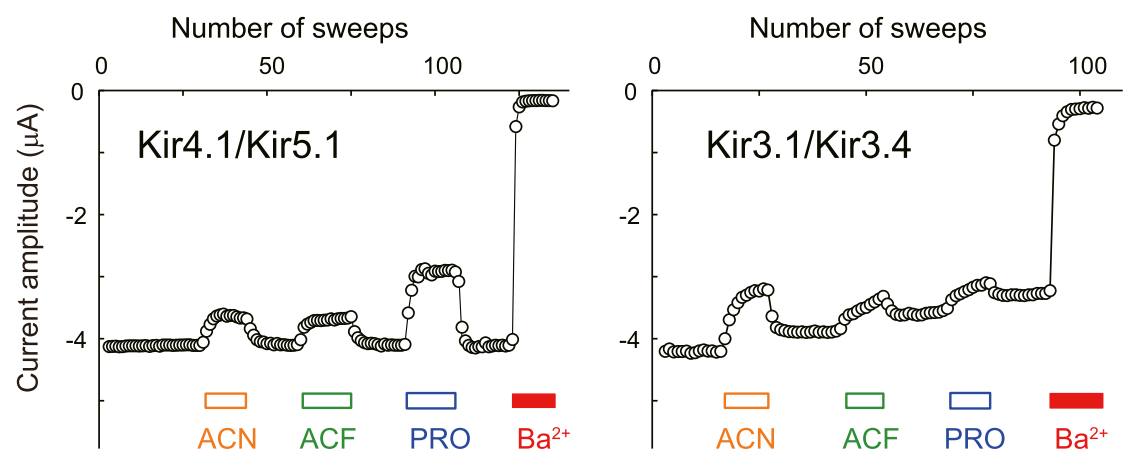

Number of sweeps
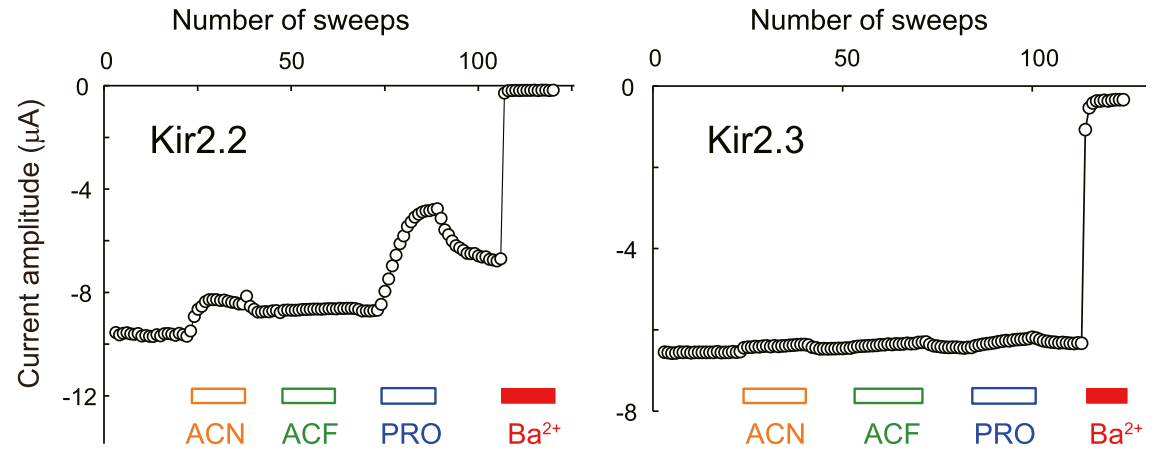

Fig. 3. Current blockage of Kir channels by proflavine derivatives. Whole-cell currents recorded in oocytes expressing Kir4.1/Kir5.1, Kir3.1/Kir3.4, Kir2.2, or Kir2.3 were measured using two-electrode voltage clamp (A). Combined step pulses, as shown in Fig. 1, were delivered every 3 seconds and current amplitude was recorded at the end of the hyperpolarized potential (B). Acrinol (ACN), acriflavine (ACF), and proflavine (PRO) were perfused at a concentration of $100 \mu \mathrm{M}$, as indicated by the bars in each panel. $\mathrm{Ba}^{2+}$ was also administrated at the end of each experiment to estimate the leak current amplitude at the hyperpolarized membrane potentials.

Kir3.1 and Kir3.4, and Kir3.2), an ATP-sensitive Kir channel (Kir6.2 $\Delta 36$ ), and $\mathrm{K}^{+}$-transporter Kir channels (Kir4.1, Kir4.2, Kir4.1 and Kir5.1, and Kir7.1). We administered acrinol, acriflavine, and proflavine serially to the cells and measured the $\mathrm{K}^{+}$current by applying combined-voltage step pulses. Typical traces and current responses of the Kir channels to these chemicals are shown in Fig. 3 and Supplemental Fig. 3.

The proflavine derivatives blocked the channels to some extent, and the pattern of current inhibition could be classified into three groups: 1) Kir1.1-type inhibition (rapid turn on and turn off of blockage, reversible suppression), 2) Kir3.2-type inhibition (slow turn on and turn off of blockage, sustained suppression), and 3) no inhibition. The heteromeric Kir4.1/Kir5.1 channel was the only subunit combination that was suppressed by all three proflavine analogs in a similar way to Kir1.1 (Fig. 3; Table 1). However, while acrinol strongly inhibited Kir1.1, Kir4.1/Kir5.1 was more sensitive to proflavine.

In contrast to Kir1.1-type inhibition, Kir3.2-type inhibition developed slowly (Fig. 1). Therefore, brief perfusion of the compounds led to incomplete blockage of this type of Kir channels. Nevertheless, these results provided information on how Kir channels are sensitive to proflavine analogs qualitatively: of the four homomeric strong inward rectifiers, Kir2.2 was unique in its susceptibility to the proflavine analogs, and was effectively suppressed by proflavine (Fig. 3). Kir6.2A36 and Kir4.2 (Supplemental Fig. 3), as well as the heteromeric G protein-gated Kir3.x channels, were also sensitive to the compounds. The other Kir channels, Kir2.1, Kir2.3, Kir2.4, Kir4.1, and Kir7.1, were essentially insensitive to the compounds tested. Like polyamines and $\mathrm{Mg}^{2+}$, which confer inward rectification property on Kir channels (Hibino et al., 2010), proflavine could be speculated to enter the central cavity of Kir3.2 from the intracellular side and prevent outward current (Kawada et al., 2016). Selective blockage of Kir2.2 among Kir2.x subfamily members implicated that the mechanism of current inhibition by proflavine and its derivatives is distinct from those by polyamines and $\mathrm{Mg}^{2+}$. On the other hand, the manner of current

TABLE 1

Kir channel subunits have different sensitivity to proflavine analogs $\mathrm{Ba}^{2+}$-sensitive $\mathrm{K}^{+}$currents were recorded from oocytes expressing various combinations of Kir channel subunits as in Fig. 3. The current amplitude recorded 1 minute after application of compounds (acrinol, acriflavine, or proflavine at a concentration of $100 \mu \mathrm{M}$ ) was divided by that in the absence of the compounds. Numbers in italic type represent members that exhibited Kir3.2-type slow inhibition and numbers in parenthesis represent members that were faint to judge whether Kir1.1-type fast inhibition or Kir3.2-type slow inhibition occurred. The number of examinations is indicated by $n$.

\begin{tabular}{lcccr}
\hline $\begin{array}{c}\text { Subunit } \\
\text { Composition }\end{array}$ & Acrinol & Acriflavine & Proflavine & $n$ \\
\hline Kir1.1 & $52.2 \pm 3.3$ & $13.0 \pm 1.1$ & $8.9 \pm 0.8$ & 10 \\
Kir2.1 & $(0.5 \pm 0.2)$ & $(0.2 \pm 0.1)$ & $(0.4 \pm 0.2)$ & 7 \\
Kir2.2 & $24.5 \pm 2.5$ & $10.0 \pm 2.2$ & $62.4 \pm 9.9$ & 9 \\
Kir2.3 & $(1.7 \pm 0.4)$ & $(1.9 \pm 0.4)$ & $(2.8 \pm 0.5)$ & 8 \\
Kir2.4 & $(1.2 \pm 0.5)$ & $(0.8 \pm 0.2)$ & $(0.6 \pm 0.3)$ & 11 \\
Kir3.1/Kir3.2c & $8.1 \pm 0.8$ & $12.4 \pm 3.0$ & $19.0 \pm 3.0$ & 9 \\
Kir3.1/Kir3.4 & $25.8 \pm 1.7$ & $18.5 \pm 1.9$ & $20.5 \pm 3.6$ & 8 \\
Kir3.2d & $9.9 \pm 3.5$ & $11.7 \pm 1.5$ & $19.1 \pm 1.8$ & 15 \\
Kir4.1 & $(1.7 \pm 0.8)$ & $(1.6 \pm 0.5)$ & $(2.8 \pm 1.1)$ & 12 \\
Kir4.1/Kir5.1 & $10.7 \pm 1.6$ & $7.0 \pm 0.9$ & $33.3 \pm 8.1$ & 11 \\
Kir4.2 & $15.3 \pm 1.0$ & $13.6 \pm 2.0$ & $17.0 \pm 5.3$ & 8 \\
Kir6.2 36 & $13.9 \pm 2.0$ & $17.8 \pm 3.6$ & $30.8 \pm 5.0$ & 10 \\
Kir7.1 & $(0.2 \pm 0.1)$ & $(0.1 \pm 0.1)$ & $(0.3 \pm 0.1)$ & 10 \\
\hline
\end{tabular}


A

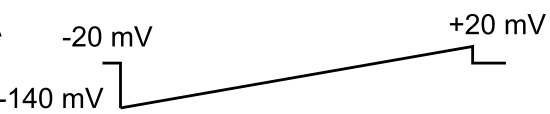

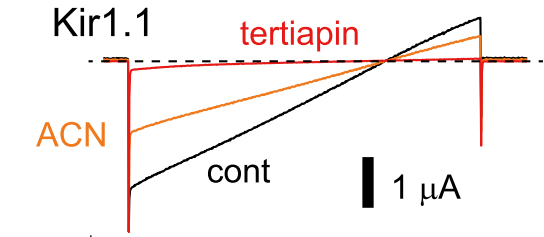
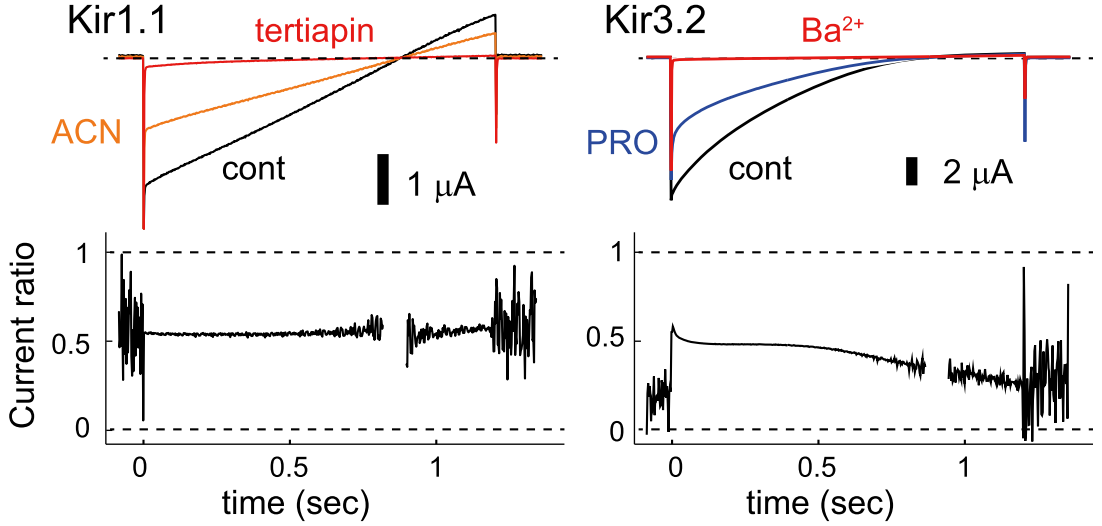

Kir4.1/Kir5.1
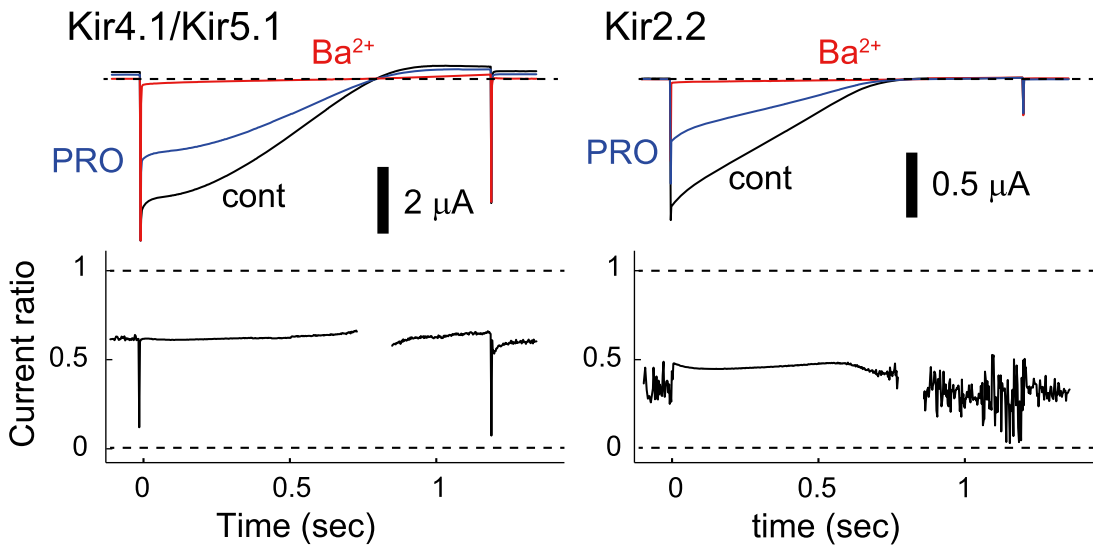

B

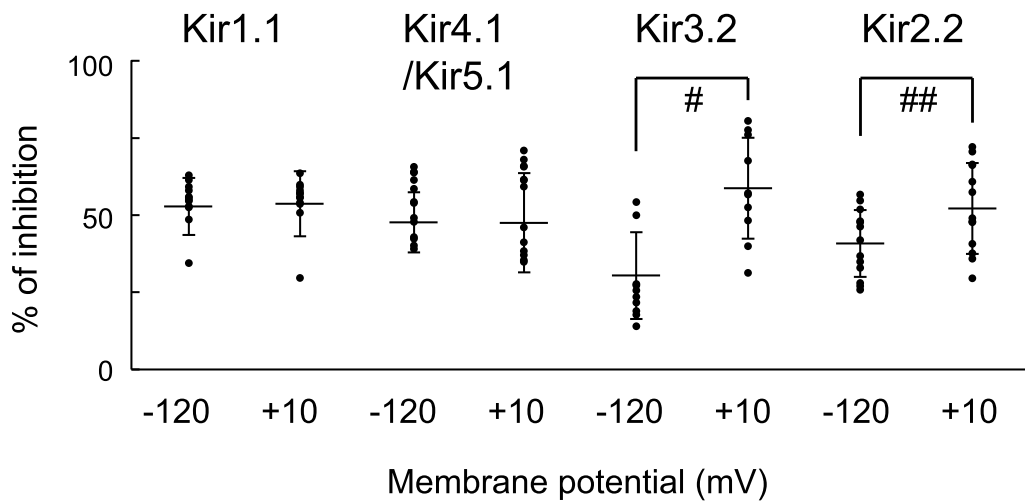

Fig. 4. Voltage dependence of current blockage by proflavine derivatives. (A) Currents elicited from oocytes expressing Kir1.1, Kir3.2, Kir4.1/Kir5.1, or Kir2.2. The oocytes were held at $-20 \mathrm{mV}$, and a linear voltage ramp from -140 to $+20 \mathrm{mV}$ for 1.2 seconds was repeatedly applied. Leak current levels were estimated by the perfusion of tertiapin for Kir1.1-expressing cells and $\mathrm{Ba}^{2+}$ for cells expressing Kir3.2, Kir4.1/Kir5.1, or Kir2.2 (see Materials and Methods). The $\mathrm{K}^{+}$current in the presence of $100 \mu \mathrm{M}$ acrinol [(ACN); Kir1.1] or proflavine [(PRO); Kir3.2, Kir4.1/Kir5.1, and Kir2.2] was divided by the current measured in their absence. The current ratio calculated around the reversal potential was omitted. (B) Effects of membrane potential on current inhibition by proflavine derivatives. The percentages of current inhibition measured at -120 and $+10 \mathrm{mV}$ were calculated by dividing the currents recorded in the presence and absence of the proflavine derivatives. Data are shown as mean \pm S.D. $(n=10$ 13); \# and \#\# indicate significance at $P<0.0001$ and $P<0.001$, respectively. inhibition of Kir1.1 and Kir4.1/Kir5.1 are unique among Kir channel members.

Voltage-Dependent and -Independent Current Blockage by Proflavine Analogs. Proflavine inhibited the outward current of Kir3.2 much more than its inward current (Kawada et al., 2016). To test the voltage dependency of Kir1.1 blockage by proflavine, $\mathrm{K}^{+}$currents were repeatedly generated by a ramp pulse from -140 to $+20 \mathrm{mV}$, and then by the serially applied proflavine analog, acrinol $(100 \mu \mathrm{M})$, and tertiapin (Fig. 4A). Acrinol inhibited Kir1.1 at all membrane potentials. When dividing the tertiapin-sensitive current in the presence of acrinol by that in its absence, we found the current ratio was almost constant during the ramp pulse. The inhibition of Kir1.1 by acrinol was $52.8 \% \pm 11.0 \%$ at $-120 \mathrm{mV}$ and $53.1 \% \pm 12.3 \%$ at $+10 \mathrm{mV}(n=12)$ (Fig. $4 \mathrm{~B})$, indicating that this inhibition is independent of membrane potential. The current of the Kir4.1/Kir5.1 complex was also decreased by proflavine, and again there were no significant differences in the extent of inhibition at different membrane potentials: inhibition was $47.7 \% \pm 9.7 \%$ at $-120 \mathrm{mV}$ and $47.2 \% \pm 18.7 \%$ 
A

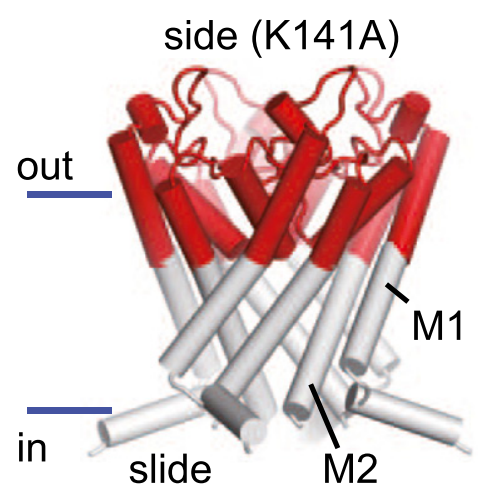

B

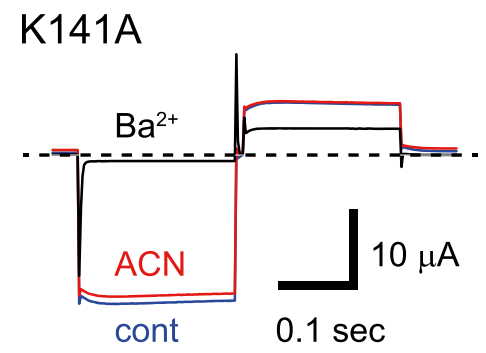

K141B
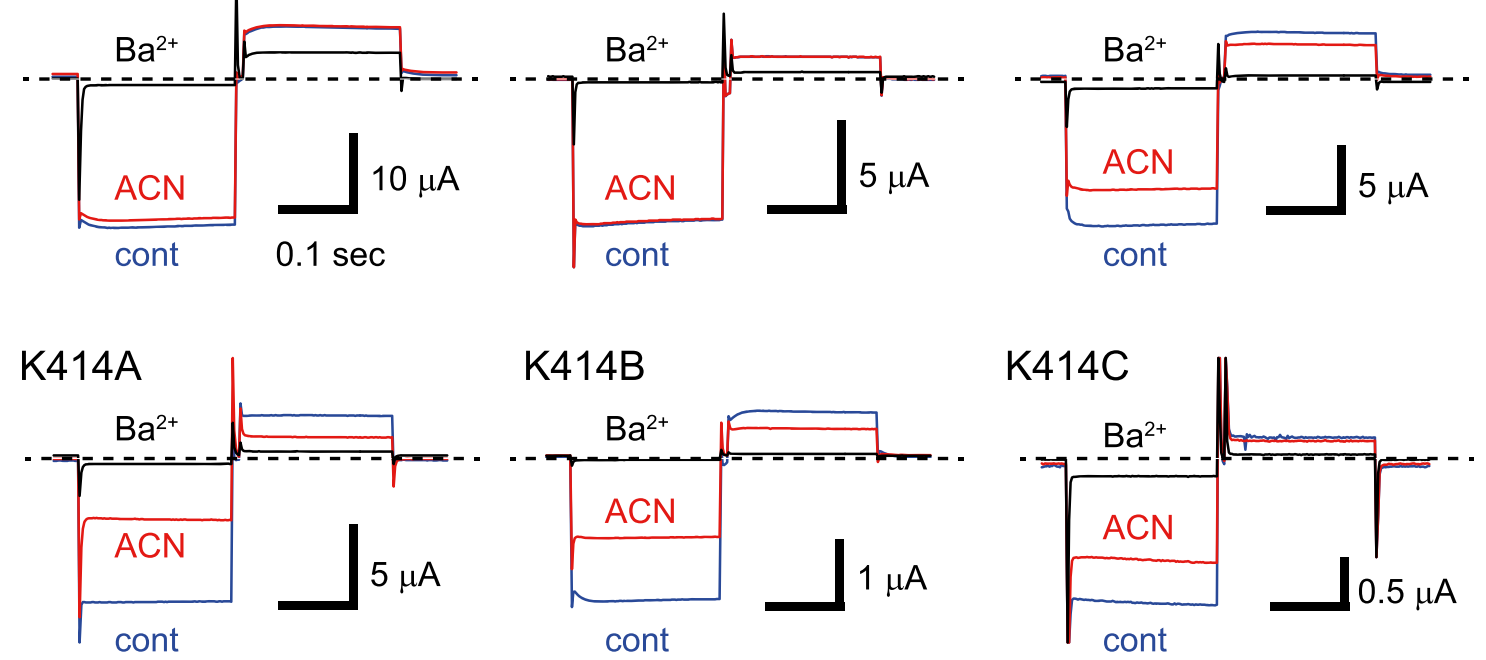

C

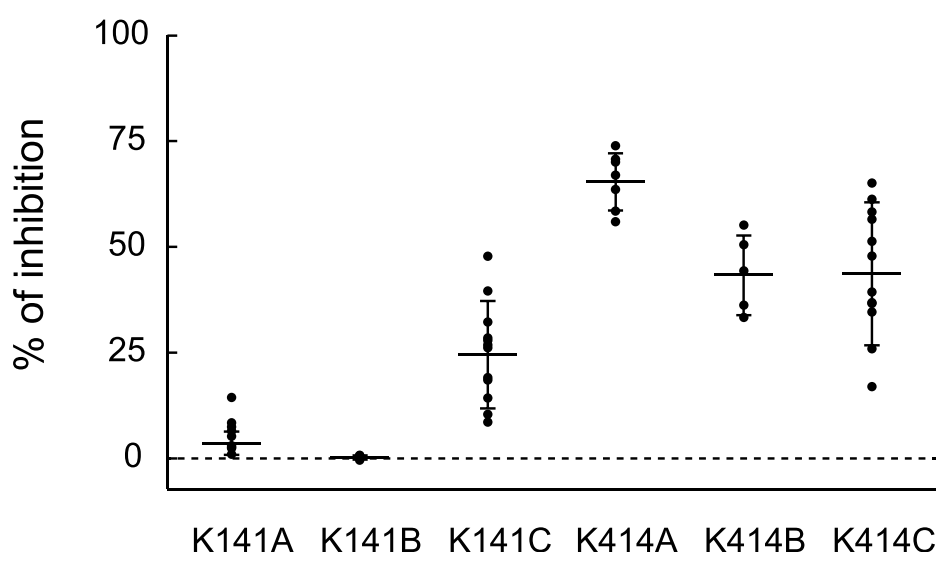

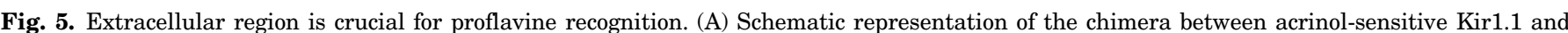

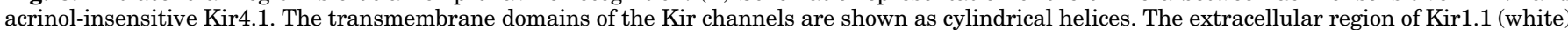

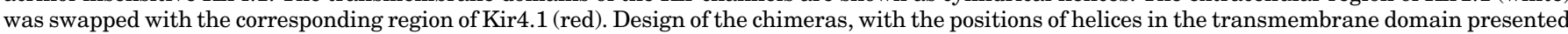

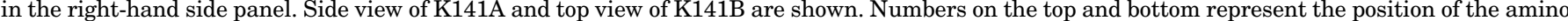

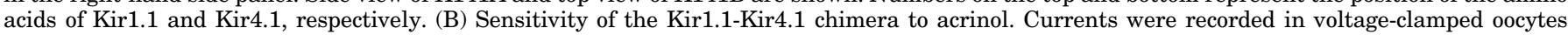


A

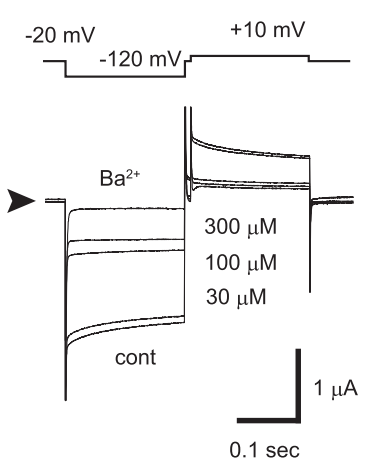

B

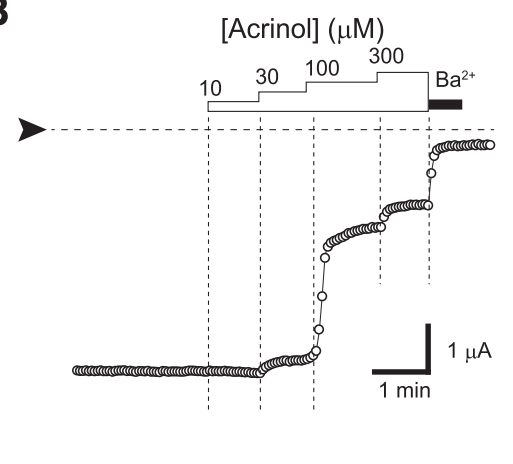

C

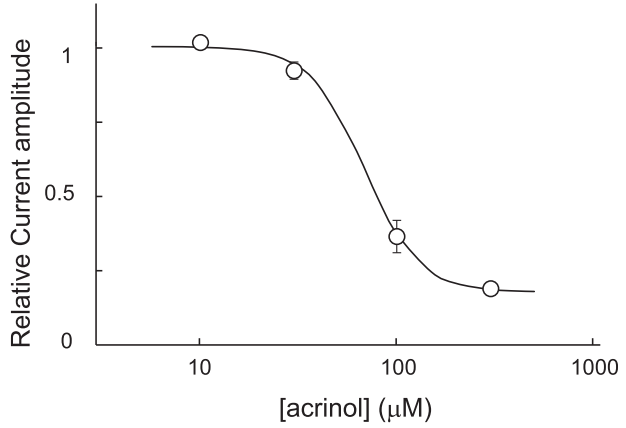

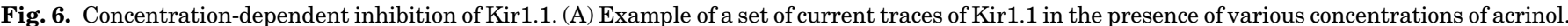

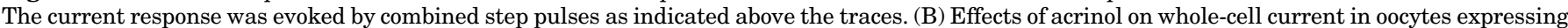

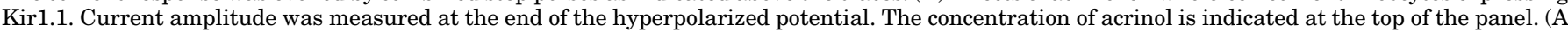

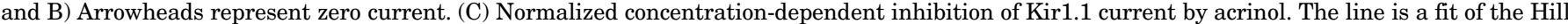
equation to the data, as discussed in Materials and Methods, with the $\mathrm{IC}_{50}$ value of $68.5 \mu \mathrm{M}$ and Hill coefficient of 3.09 .

at $+10 \mathrm{mV}(n=13)$. Therefore, blockage of the heteromeric Kir4.1/Kir5.1 channel also appears to be independent of membrane potential.

Proflavine also blocked Kir3.2 and Kir2.2 at all membrane potentials, but dividing the $\mathrm{K}^{+}$current in the presence of proflavine by the total $\mathrm{K}^{+}$current revealed that the blockage was greater at depolarized membrane potentials. Furthermore, directly after the jump to hyperpolarization from the holding potential, transient recovery from the blockage was recorded in oocytes expressing both channels. This may have been caused by the transient relief from current inhibition by rapid voltage shift and the delayed suppression under equilibrium at hyperpolarization (Kawada et al., 2016). The percentages of inhibition were $30.5 \% \pm 14.4 \%$ at $-120 \mathrm{mV}$ and $58.3 \% \pm 16.3 \%$ at $+10 \mathrm{mV}$ for Kir3.2 $(n=10)$ and $40.6 \%$ $\pm 10.8 \%$ at $-120 \mathrm{mV}$ and $52.0 \% \pm 14.7 \%$ at $+10 \mathrm{mV}$ for Kir2.2 $(n=13)$. Therefore, proflavine-induced blockage of Kir3.2 and Kir2.2 was dependent on voltage. These two Kir channels demonstrated slow development and imperfect recovery of current suppression. These drug actions were also observed in Kir3.1/Kir3.2, Kir3.1/Kir3.4, Kir4.2, and Kir6.2A36. Since voltage-dependent blockage is one of the features of proflavine as a pore blocker (Kawada et al., 2016), Kir3.2-type inhibition may indicate that the chemical accesses the central cavity from the intracellular side. In conclusion, the manner of Kir1.1- and Kir3.2-type inhibition may be explained, at least in part, by the difference in the mode of drug binding; specifically, whether the drug binds in the central cavity or not.

Acrinol Associates with the Extracellular Region of Kir1.1. Based on the rapid onset and recovery of block of Kir1.1 (Fig. 1A), the extracellular portion of the channels was assumed to mediate the action of proflavine analogs. We swapped the extracellular region of proflavine-sensitive Kir1.1 with the corresponding region of proflavineinsensitive Kir4.1 (Fig. 5A). This chimera (K141A) did not show the same degree of susceptibility to acrinol (inhibition: $3.7 \% \pm 3.1 \%, n=8$ ) (Fig. $5, \mathrm{~B}$ and $\mathrm{C}$ ). In contrast, the reverse chimera (K414A) was sensitive to acrinol (inhibition: $65 \% \pm 8 \%$, $n=7$ ). This clearly suggests that the outward-facing region mediates the action of acrinol.

The substituted region consists of the slide helix and selectivity filter with the termini of M1 and M2 helices. When dividing this portion at the beginning of the slide helix into two segments, the latter (from the slide helix to the M2 helix) exclusively occupies the subunit interface of the extracellular portion and the selectivity filter (Fig. 5A). To gain insight into the structural element responsible for acrinol-induced current blockage, we produced chimeras by placing each of these short segments of Kir4.1 into the equivalent region of Kir1.1 (K141B and $\mathrm{K} 141 \mathrm{C}$ ), and we also prepared chimeras in which the Nand C-termini of Kir4.1 flanked the short Kir1.1 segments (K414B and K414C). K141B was insensitive to acrinol (inhibition: $0.3 \% \pm 0.5 \%, n=6$ ), but $\mathrm{K} 141 \mathrm{C}$ was moderately blocked by it (inhibition: $25 \% \pm 14.3 \%, n=12$ ). In contrast, acrinol inhibited both K414B and K414C (K414B: $44 \% \pm 11 \%$, $n=5$; K414C: $44 \% \pm 20 \%, n=12$ ). These results suggest that acrinol is recognized by multiple residues present on the extracellular region, and neither amino acids close to the selectivity filter nor the interface between subunits are prerequisite for the recognition by themselves. It also implicates that the compound-binding site is located at each subunit.

Concentration-Dependent Current Inhibition by Acrinol. To gain further understanding of the binding mode, we recorded the Kir1.1 current in various concentrations of acrinol (Fig. 6). The current decreased in a concentrationdependent manner, with an $\mathrm{IC}_{50}$ value of $68.5 \pm 18.1 \mu \mathrm{M}$ and a Hill coefficient of $3.09 \pm 0.64(n=8)$. A possible implication of the Hill coefficient is that at least three compounds were required to block the channel. This agrees with the idea that each subunit possesses a putative compound-binding site (Fig. 5).

According to the open large-scale bioactivity database by the European Molecular Biology Laboratory (ChEMBL) (https:// www.ebi.ac.uk/chembl/) (Bento et al., 2014), acrinol has a $\mathrm{p} K_{\mathrm{a}}$ value of 11.22. This indicates that most acrinol are positively charged in neutral $\mathrm{pH}$ conditions. Kir1.1 is sensitive to internal $\mathrm{pH}$, but insensitive to external $\mathrm{pH}$ (Tsai et al., 1995). Next, we tested whether external $\mathrm{pH}$ might affect the 

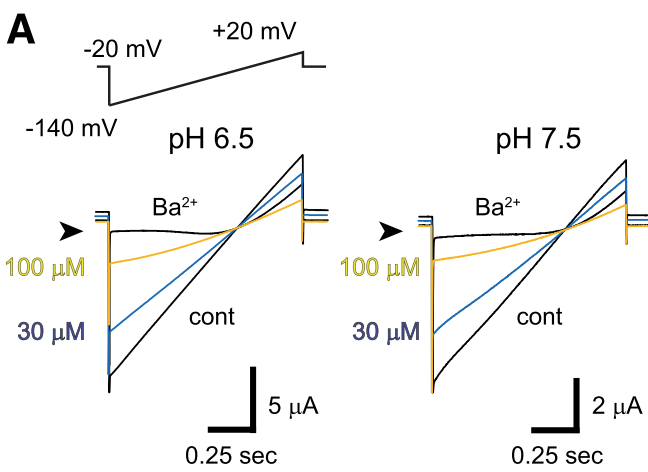

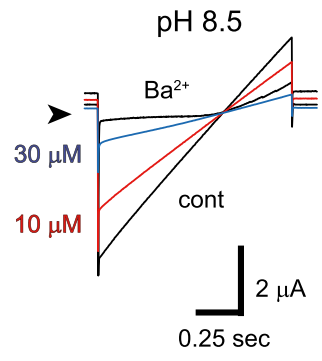

B

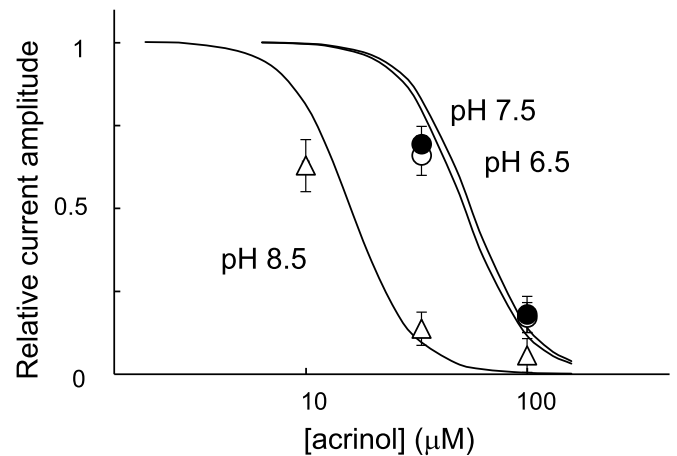

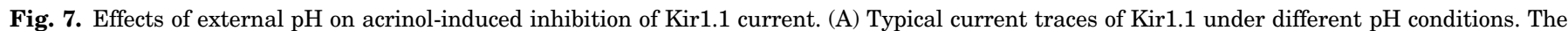

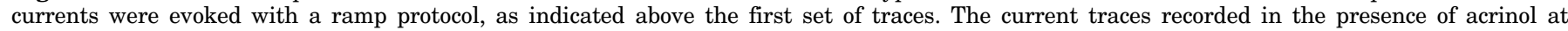

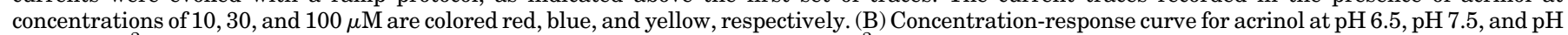
8.5. The $\mathrm{Ba}^{2+}$-sensitive current amplitude at $-120 \mathrm{mV}$ was normalized to total $\mathrm{Ba}^{2+}$-sensitive current.

blockage of Kir1.1 by acrinol (Fig. 7). Acrinol inhibited Kir1.1 in a dose-dependent manner in all $\mathrm{pH}$ conditions examined (pH 6.5, 7.5, and 8.5). When fitting the responses with a Hill coefficient of 3.09 (Fig. 6), the $\mathrm{IC}_{50}$ values for acrinol were calculated as $50 \pm 44 \mu \mathrm{M}$ at pH $6.5(n=6), 53 \pm 37 \mu \mathrm{M}$ at $\mathrm{pH}$ $7.5(n=7)$, and $15.3 \pm 14.5 \mu \mathrm{M}$ at pH $8.5(n=7)$. The degree of current inhibition of Kir 1.1 by acrinol at $\mathrm{pH} 6.5$ was almost the same as that at $\mathrm{pH}$ 7.5. However, the dose-response curve recorded at $\mathrm{pH} 8.5$ shifted leftward relative to the curve recorded at $\mathrm{pH}$ 7.5. Therefore, it seems likely that the ionization state of Kir1.1 and/or the molecules around it that face the external solution affects ligand recognition.

\section{Discussion}

Tetrameric and pseudo-tetrameric cation channels are the target of remedies for various pathologic conditions. Many compounds enter the ion conduction pathway from the intracellular side and bind to the central cavity of the channels located in the membrane plane. Previously, we reported that proflavine and its derivatives were pore blockers of Kir3.2, and that this inhibition was characterized by slow onset and recovery, and by sustained and voltage-dependent blockage (Kawada et al., 2016). Kir2.2, Kir3.x, Kir4.2, and Kir6.2s36 responded similarly to proflavine and its analogs (Figs. 3 and 4; Table 1). Thus, the derivatives appear to act as pore blockers of these Kir channels. However, proflavine blockage of Kir1.1 functioned uniquely, with high sensitivity to extracellular compounds (Figs. 1 and 3 ) but less sensitivity to membrane potential (Fig. 4). We also found that the extracellular region of the channel was crucial for drug sensitivity (Fig. 5), and the extracellular $\mathrm{pH}$ milieu affected the response to the compound (Fig. 7). These results implied that proflavine derivatives associate with the extracellular portion of Kir1.1. This type of inhibition was also observed with the heteromeric Kir4.1/Kir5.1 channel. Thus, a limited number of Kir channels enable access by proflavine derivatives from the extracellular side.

The central cavity is not the only place where Kir channel blockers interact. Binding of tertiapin at the external vestibule of the ion conduction pathway prevents the ionic flow of a subset of Kir channel members (Jin and Lu, 1998). Kir channels also possess an ion-permeable pore in a domain exposed to the cytoplasm, which connects to the pore within the transmembrane domain (Nishida and MacKinnon, 2002). The cytoplasmic pore of Kir2.1 reportedly binds the antimalarial agent chloroquine, leading to current inhibition (Rodríguez-Menchaca et al., 2008). Furthermore, chloroquine appears to block the channels by interacting with several distinct areas: the central cavities of Kir6.2 and Kir4.1 (PonceBalbuena et al., 2012; Marmolejo-Murillo et al., 2017) and the $\mathrm{PIP}_{2}$-binding site of Kir6.2 (Ponce-Balbuena et al., 2012). These observations indicate that the binding of molecules with an affinity for any location within the ion conduction pathway, or the prevention of the association of activator compounds, would lead to a blockage of the ion passage through the pore. Therefore, proflavine derivatives have the capacity to differentially block the channel by associating with the extracellular portion of Kir1.1.

How does the compound association in the extracellular region cause current blockage? Wang et al. (2016) reported that there is less conformation change in the corresponding part of the bacterial Kir-channel homolog during gating. Fragmentation of the extracellular portion suggested that multiple residues were involved in the drug recognition (Fig. 5 ). Furthermore, based on the symmetric property of the channel, the compound-binding site might be located at each subunit. Therefore, even though the portion exposed to the external fluid is restricted the area certainly alters their conformation during gating, and binding may stabilize the closed conformation of the channels.

Structural transition in Kir channels during gating is not limited to the transmembrane domain. The cytoplasmic domain is thought to rotate (Riven et al., 2003) and change distance (Tao et al., 2009) relative to the transmembrane domain. Reorientations of the subunit interface (Clarke et al., 2010) and changes in the conformation of each subunit (Inanobe et al., 2011a) are also plausible. Furthermore, the cytoplasmic pore is required to dilate in order to allow $\mathrm{K}^{+}$ permeation (Robertson et al., 2008; Inanobe et al., 2011b). Therefore, global alteration in the conformation of the entire molecule may underlie Kir-channel functioning. This is in accordance with the distribution of amino acids responsible for the small-molecule activators of Kir3.x channels, i.e., sodium (Ho and Murrell-Lagnado, 1999) and alcohol (Aryal et al., 
2009), associate with the cytoplasmic domain of Kir3.2 and Kir3.4, and $\mathrm{PIP}_{2}$ interacting at the interface between the transmembrane and cytoplasmic domains of all Kir-channel members (Kobrinsky et al., 2000). Furthermore, it has been suggested that a small compound, ML297, recently identified as a Kir3.1-specific activator (Kaufmann et al., 2013), interacts with two amino acids located in the transmembrane domain (Wydeven et al., 2014). These observations reveal that chemicals binding to any position on the channels may facilitate alteration of their activity by modulating structural transition negatively or positively. It is thought that a compound that binds to a site distinct from the primary binding site of blockers has the potential to overcome some of the drawbacks of current chemicals (Imming et al., 2006). While Kir channels exhibited multiple modes of proflavine recognition, the interactions between their extracellular portions and proflavine derivatives were selective. Since it is reasonable to assume that all Kir channels share a comparable molecular architecture and manner of conformational transition, the difference in the drug sensitivity is based on the difference in amino acids at drug recognition sites. Identifying the mode of binding might shed light on the design of the subunit-selective modulators of Kir channels.

\section{Acknowledgments}

The authors thank Miho Kawabata and Kiyoko Yoshimoto for technical assistance, and Rika Okuya for secretarial support.

\section{Authorship Contributions}

Participated in research design: Inanobe.

Conducted experiments: Inanobe, Itamochi.

Performed data analysis: Inanobe, Itamochi.

Wrote or contributed to the writing of the manuscript: Inanobe, Kurachi.

\section{References}

Aryal P, Dvir H, Choe S, and Slesinger PA (2009) A discrete alcohol pocket involved in GIRK channel activation. Nat Neurosci 12:988-995.

Bento AP, Gaulton A, Hersey A, Bellis LJ, Chambers J, Davies M, Krüger FA, Light Y, Mak L, McGlinchey S, et al. (2014) The ChEMBL bioactivity database: an update. Nucleic Acids Res 42:D1083-D1090.

Bhave G, Chauder BA, Liu W, Dawson ES, Kadakia R, Nguyen TT, Lewis LM, Meiler J, Weaver CD, Satlin LM, et al. (2011) Development of a selective small-molecule inhibitor of Kir1.1, the renal outer medullary potassium channel. Mol Pharmacol 79:42-50.

Bichet D, Haass FA, and Jan LY (2003) Merging functional studies with structures of inward-rectifier $\mathrm{K}^{+}$channels. Nat Rev Neurosci 4:957-967.

Clarke OB, Caputo AT, Hill AP, Vandenberg JI, Smith BJ, and Gulbis JM (2010) Domain reorientation and rotation of an intracellular assembly regulate conduction in Kir potassium channels. Cell 141:1018-1029.

Hebert SC (2003) Bartter syndrome. Curr Opin Nephrol Hypertens 12:527-532.

Hebert SC, Desir G, Giebisch G, and Wang W (2005) Molecular diversity and regulation of renal potassium channels. Physiol Rev 85:319-371.

Hibino H, Inanobe A, Furutani K, Murakami S, Findlay I, and Kurachi Y (2010) Inwardly rectifying potassium channels: their structure, function, and physiological roles. Physiol Rev 90:291-366.

Ho IH and Murrell-Lagnado RD (1999) Molecular mechanism for sodium-dependent activation of $\mathrm{G}$ protein-gated $\mathrm{K}^{+}$channels. $J$ Physiol 520:645-651.

Ho K, Nichols CG, Lederer WJ, Lytton J, Vassilev PM, Kanazirska MV, and Hebert SC (1993) Cloning and expression of an inwardly rectifying ATP-regulated potassium channel. Nature 362:31-38.

Imming P, Sinning C, and Meyer A (2006) Drugs, their targets and the nature and number of drug targets. Nat Rev Drug Discov 5:821-834.

Inanobe A, Horio Y, Fujita A, Tanemoto M, Hibino H, Inageda K, and Kurachi Y (1999a) Molecular cloning and characterization of a novel splicing variant of the Kir3.2 subunit predominantly expressed in mouse testis. $J$ Physiol 521:19-30.

Inanobe A, Matsuura T, Nakagawa A, and Kurachi Y (2011a) Inverse agonist-like action of cadmium on G-protein-gated inward-rectifier $\mathrm{K}^{+}$channels. Biochem Biophys Res Commun 407:366-371.

Inanobe A, Morishige KI, Takahashi N, Ito H, Yamada M, Takumi T, Nishina H, Takahashi K, Kanaho Y, Katada T, et al. (1995) $\mathrm{G}_{\beta \gamma}$ directly binds to the carboxyl terminus of the $\mathrm{G}$ protein-gated muscarinic $\mathrm{K}^{+}$channel, GIRK1. Biochem Biophys Res Commun 212:1022-1028.

Inanobe A, Nakagawa A, and Kurachi Y (2011b) Interactions of cations with the cytoplasmic pores of inward rectifier $\mathrm{K}^{+}$channels in the closed state. $J$ Biol Chem 286:41801-41811.
Inanobe A, Yoshimoto Y, Horio Y, Morishige KI, Hibino H, Matsumoto S, Tokunaga Y, Maeda T, Hata Y, Takai Y, et al. (1999b) Characterization of G-protein-gated $\mathrm{K}^{+}$ channels composed of Kir3.2 subunits in dopaminergic neurons of the substantia nigra. J Neurosci 19:1006-1017.

Jin W and Lu Z (1998) A novel high-affinity inhibitor for inward-rectifier $\mathrm{K}^{+}$channels. Biochemistry 37:13291-13299.

Kaufmann K, Romaine I, Days E, Pascual C, Malik A, Yang L, Zou B, Du Y, Sliwosk G, Morrison RD, et al. (2013) ML297 (VU0456810), the first potent and selective activator of the GIRK potassium channel, displays antiepileptic properties in mice. ACS Chem Neurosci 4:1278-1286.

Kawada H, Inanobe A, and Kurachi Y (2016) Isolation of proflavine as a blocker of G protein-gated inward rectifier potassium channels by a cell growth-based screening system. Neuropharmacology 109:18-28.

Kobrinsky E, Mirshahi T, Zhang H, Jin T, and Logothetis DE (2000) Receptormediated hydrolysis of plasma membrane messenger $\mathrm{PIP}_{2}$ leads to $\mathrm{K}^{+}$-current desensitization. Nat Cell Biol 2:507-514.

Kondo C, Isomoto S, Matsumoto S, Yamada M, Horio Y, Yamashita S, TakemuraKameda K, Matsuzawa Y, and Kurachi Y (1996) Cloning and functional expression of a novel isoform of ROMK inwardly rectifying ATP-dependent $\mathrm{K}^{+}$channel, ROMK6 (Kir1.1f). FEBS Lett 399:122-126.

Krapivinsky G, Gordon EA, Wickman K, Velimirović B, Krapivinsky L, and Clapham $\mathrm{DE}$ (1995) The G-protein-gated atrial $\mathrm{K}^{+}$channel $\mathrm{I}_{\mathrm{KACh}}$ is a heteromultimer of two inwardly rectifying $\mathrm{K}^{+}$-channel proteins. Nature $374: 135-141$.

Lewis LM, Bhave G, Chauder BA, Banerjee S, Lornsen KA, Redha R, Fallen K, Lindsley CW, Weaver CD, and Denton JS (2009) High-throughput screening reveals a small-molecule inhibitor of the renal outer medullary potassium channel and Kir7.1. Mol Pharmacol 76:1094-1103.

Lorenz JN, Baird NR, Judd LM, Noonan WT, Andringa A, Doetschman T, Manning PA, Liu LH, Miller ML, and Shull GE (2002) Impaired renal $\mathrm{NaCl}$ absorption in mice lacking the ROMK potassium channel, a model for type II Bartter's syndrome. J Biol Chem 277:37871-37880.

Marmolejo-Murillo LG, Aréchiga-Figueroa IA, Moreno-Galindo EG, Navarro-Polanco RA, Rodríguez-Menchaca AA, Cui M, Sánchez-Chapula JA, and Ferrer T (2017) Chloroquine blocks the Kir4.1 channels by an open-pore blocking mechanism. Eur J Pharmacol 800:40-47.

Morishige K, Takahashi N, Jahangir A, Yamada M, Koyama H, Zanelli JS, and Kurachi Y (1994) Molecular cloning and functional expression of a novel brainspecific inward rectifier potassium channel. FEBS Lett 346:251-256.

Nishida M and MacKinnon R (2002) Structural basis of inward rectification: cytoplasmic pore of the G protein-gated inward rectifier GIRK1 at 1.8 A resolution. Cell 111:957-965.

Ponce-Balbuena D, Rodríguez-Menchaca AA, López-Izquierdo A, Ferrer T, Kurata HT, Nichols CG, and Sánchez-Chapula JA (2012) Molecular mechanisms of chloroquine inhibition of heterologously expressed Kir6.2/SUR2A channels. Mol Pharmacol 82:803-813.

Riven I, Kalmanzon E, Segev L, and Reuveny E (2003) Conformational rearrangements associated with the gating of the $\mathrm{G}$ protein-coupled potassium channel revealed by FRET microscopy. Neuron 38:225-235.

Robertson JL, Palmer LG, and Roux B (2008) Long-pore electrostatics in inwardrectifier potassium channels. J Gen Physiol 132:613-632.

Rodríguez-Menchaca AA, Navarro-Polanco RA, Ferrer-Villada T, Rupp J, Sachse FB, Tristani-Firouzi M, and Sánchez-Chapula JA (2008) The molecular basis of chloroquine block of the inward rectifier Kir2.1 channel. Proc Natl Acad Sci USA 105:1364-1368.

Sanguinetti MC, Jiang C, Curran ME, and Keating MT (1995) A mechanistic link between an inherited and an acquired cardiac arrhythmia: $H E R G$ encodes the $\mathrm{I}_{\mathrm{K}_{r}}$ potassium channel. Cell 81:299-307.

Sica DA (2011) Diuretic use in renal disease. Nat Rev Nephrol 8:100-109.

Takahashi N, Morishige K, Jahangir A, Yamada M, Findlay I, Koyama H, and Kurachi Y (1994) Molecular cloning and functional expression of cDNA encoding a second class of inward rectifier potassium channels in the mouse brain. $J$ Biol Chem 269:23274-23279.

Takumi T, Ishii T, Horio Y, Morishige K, Takahashi N, Yamada M, Yamashita T, Kiyama H, Sohmiya K, Nakanishi S, et al. (1995) A novel ATP-dependent inward rectifier potassium channel expressed predominantly in glial cells. J Biol Chem 270:16339-16346.

Tamargo J, Segura J, and Ruilope LM (2014) Diuretics in the treatment of hypertension. Part 2: loop diuretics and potassium-sparing agents. Expert Opin Pharmacother 15:605-621.

Tao X, Avalos JL, Chen J, and MacKinnon R (2009) Crystal structure of the eukaryotic strong inward-rectifier $\mathrm{K}^{+}$channel Kir2.2 at $3.1 \AA$ resolution. Science 326:1668-1674.

Tsai TD, Shuck ME, Thompson DP, Bienkowski MJ, and Lee KS (1995) Intracellular $\mathrm{H}^{+}$inhibits a cloned rat kidney outer medulla $\mathrm{K}^{+}$channel expressed in Xenopus oocytes. Am J Physiol 268:C1173-C1178.

Wang S, Vafabakhsh R, Borschel WF, Ha T, and Nichols CG (2016) Structural dynamics of potassium-channel gating revealed by single-molecule FRET. Nat Struct Mol Biol 23:31-36.

Wydeven N, Marron Fernandez de Velasco E, Du Y, Benneyworth MA, Hearing MC, Fischer RA, Thomas MJ, Weaver CD, and Wickman K (2014) Mechanisms underlying the activation of G-protein-gated inwardly rectifying $\mathrm{K}^{+}$(GIRK) channels by the novel anxiolytic drug, ML297. Proc Natl Acad Sci USA 111:10755-10760.

Address correspondence to: Atsushi Inanobe, Division of Molecular and Cellular Pharmacology, Department of Pharmacology, Graduate School of Medicine, Osaka University, 2-2 Yamada-Oka, Suita, Osaka 565-0871, Japan. E-mail: inanobe@pharma2.med.osaka-u.ac.jp; or Yoshihisa Kurachi, Division of Molecular and Cellular Pharmacology, Department of Pharmacology, Graduate School of Medicine, Osaka University, 2-2 Yamada-Oka, Suita, Osaka 565-0871, Japan. E-mail: ykurachi@pharma2.med.osaka-u.ac.jp 